\title{
See One, Do One, Teach One: A Practical Course on Methods in Mammary Gland Biology
}

\author{
Martin Jechlinger ${ }^{1} \cdot$ Howard Kendrick $^{2} \cdot$ Matthew Smalley $^{2} \cdot$ Maria dM Vivanco $^{3}$
}

Received: 15 December 2017 / Accepted: 25 December 2017 / Published online: 14 February 2018

๑) Springer Science+Business Media, LLC, part of Springer Nature 2018

The first EMBO Practical Course on "Techniques for Mammary Gland Research" took place at EMBL, Heidelberg, from the 20th - 24th March 2017. The general aim of this practical and theory course was to ensure that all participants had the opportunity to learn about and practice some of the classical assays for studying mammary gland biology and tumorigenesis and also to enjoy some of the latest state-ofthe-art technology within the imaging facilities at EMBL. Specific goals for the course were to describe, demonstrate and practice key techniques for studying human and mouse mammary gland biology, with special emphasis on discussing both systems in the context of analysis of stem/progenitor cell populations. The course hosted 20 participants from 15 different countries, providing an additional excellent opportunity for scientific exchange and networking.

\section{Day One}

Our first day's events were all located at the excellent ISG Hotel. Having made ourselves comfortable, we were briefed on how the course would work by Jacqueline Dreyer-Lamm, from EMBL. Dr. Maria Vivanco from CIC bioGUNE, Bilbao, introduced the course and its origins as an extension of the Workshop that the European Network of Breast Development and Cancer Labs (ENBDC, http://www.enbdc.org) organises every year in Weggis, Switzerland. It was the intention of this practical course to compliment the Weggis meeting, which is dedicated to oral presentations with a focus on methodology.

Maria dM Vivanco

mdmvivanco@cicbiogune.es

1 European Molecular Biology Laboratories, Heidelberg 69117, Germany

2 European Cancer Stem Cell Research Institute, Cardiff University, Cardiff CF24 4HQ, UK

3 CIC bioGUNE, Technological Park of Bizkaia, 48160 Derio, Spain
We were then fortunate enough to have Professor Nancy Hynes from the Friedrich Miescher Institute for Biomedical Research, Basel, as a guest speaker. Prof. Hynes gave an inspiring overview of her career in breast cancer research, with a particular focus on the importance of novel methodological approaches in making breakthrough discoveries.

\section{Day Two}

Day two began with a series of talks introducing the techniques associated with research into the mouse and human mammary and, more generally, FACS sorting. Professor Matthew Smalley from Cardiff University gave a brief overview of some of the mouse mammary techniques that would be covered on the course, then concentrated on the cleared fat pad transplant assay, discussing its history, its importance in the functional assessment of stem cell potential in mammary cells and its utility in other experimental approaches, such as tissue recombination experiments to test paracrine interactions.

Next, Dr. Vivanco introduced breast cancer as a highly heterogenous disease, not only among different patients, but also within each tumor. Part of this intra-tumor heterogeneity may be due to the presence of cells with stem cell properties, namely self-renewal and differentiation abilities, which allow tumor initiation and increased capacity to resist current forms of therapies. The differences in the microanatomy of human and mouse mammary gland were also discussed, as these present a challenge when selecting a model to study normal and neoplastic breast biology. Some of the most notable distinctions were discussed, for example, the fact that the mouse mammary gland lacks the dense collagenous stroma that characterises the human breast and the ducts are directly surrounded by the adipose tissue [1] Dr. Vivanco presented a detailed protocol, starting with the preparation of the corresponding ethical permits according to local regulations, for human breast tissue processing. 
Fresh reduction mammoplasty material is mechanically and enzymatically digested, followed by several centrifugation and filtration steps that are used to separate organoids and epithelial cells from adipose tissue, blood vessels and stroma. It also included variations in the basic protocol and discussion of alternative media and additives used by different laboratories with various purposes. It appears that the specific medium composition selected may depend on the question(s) being addressed.

Fluorescence-activated cell sorting (FACS) is one of the key techniques used to isolate and characterize stem cells, including cells from the mammary gland. Dr. Malte Paulsen from EMBL, Heidelberg, provided an excellent introduction to basic FACS principles, starting from best practices for defining live cells in complex tissue preparations, venturing into details about experimental design and compensation issues mostly observed with fatty tissues. Further, theory was extended into cell sorting to prepare participants for the practical part.

These presentations were followed by short talks from each of the participants, summarising in 5 min who they were, their background and their area of interest. What was particularly impressive about these presentations was not just the professionalism with which they were delivered and the diversity of participants, but also the global distribution of their home labs. Participants had come from fifteen different countries, all across Europe and as far away as India and Indonesia, providing an excellent platform for networking. An interesting observation is that there were almost 3 times more female than male applicants for the course. Most of the participants were junior researchers and therefore many of them were particularly enthusiastic about the opportunity to present their own work in the format of a short talk as well as a poster, contributing to further scientific discussions during the course.

The practical sessions (led by Prof. Smalley and Howard Kendrick, Cardiff University) began in the afternoon. Splitting the attendees into groups to enable two demonstrations to run in parallel, participants were shown how to dissect and harvest mouse mammary fat pads for both wholemount and processing to organoids or single cells for FACS or culture $[2,3]$. In parallel, the cleared mammary fat pad transplant technique was demonstrated on humanely killed mice. The requirements for adhering to local ethical and licensing regulations when operating on live anaesthetised mice was emphasised. Everyone had a chance to practice and to run through both techniques and optional or additional steps were discussed regarding processing of the samples.

For the tissue harvests, each person first wholemounted a fat pad and began the process of fixation, carmine staining, dehydration and clearing which would last the whole week, with washes and reagent changes happening periodically. The remaining fat pads were digested to organoids using two parallel protocols - one involving both mechanical (using a Tissue Chopper) and enzymatic disaggregation to organoid stage, followed by storage overnight at $4{ }^{\circ} \mathrm{C}$, and the other digesting the glands overnight at $37{ }^{\circ} \mathrm{C}$ and $5 \% \mathrm{CO}_{2}$.

For cleared fat pad transplantation, participants practiced locating the 4th mammary fat pad through a small ventral incision, freeing it from the 5th fat pad and the overlying skin, removing the portion of the gland proximal to the intramammary lymph node (which contains the endogenous epithelium in the three week old mouse) and then injecting a $10 \mu$ volume (in our case, of blue dye) into the remaining fat pad. Everyone got a chance to practice their stitching as they closed the incisions they had made, however, it was emphasised that training in basic small animal surgical techniques, such as suturing and anaesthesia, must be sought in addition to the methodology described within this course.

Prof. Smalley gave a talk on the pros and cons of FACS and in situ lineage tracing as approaches to the analysis of mammary epithelial subpopulations (Table 1) [4]. The importance of caution in data interpretation and being able to make ones experiments cross-comparable with results from other groups was emphasised. Dr. Vivanco presented the different approaches used to identify human breast stem/

Table 1 Advantages and disadvantages of FACS and lineage tracing in analysis of mammary epithelial populations

\begin{tabular}{|c|c|}
\hline FACS & Lineage Tracing \\
\hline Advantages & Advantages \\
\hline Makes no a priori assumptions & Spatial resolution \\
\hline Permits direct functional testing of isolated populations & Allows timecourse analysis \\
\hline Gene expression of populations can be tested & Enables mathematical models to be proposed \\
\hline Disadvantages & Disadvantages \\
\hline No spatial resolution & Transgenes do not necessarily replicate endogenous expression patterns \\
\hline Only a snapshot & May get different answers from use of different transgene/knock-in \\
\hline Enzymatic digestion may cleave epitopes & Depends on good microscopy \\
\hline $\begin{array}{l}\text { Enzymatic digestion and flow causes stress and may } \\
\text { change gene expression }\end{array}$ & $\begin{array}{l}\text { Cells being labelled are difficult to fully characterise (and therefore relate to } \\
\text { FACS) }\end{array}$ \\
\hline
\end{tabular}


progenitor cells, mostly based on the expression of surface antigens, such as MUC-1/CALLA, CD49f/ESA, and CD44/ $\mathrm{CD} 24$, or on functional assays, including presence of the side population, cells with increased aldehyde dehydrogenase (ALDH) activity, label-retaining capacity or ability to grow in suspension as spheres (reviewed in [5, 6]). Importantly, the presence of $\mathrm{CD} 44^{+} \mathrm{CD} 24^{-/ \mathrm{low}}$ and $\mathrm{ALDH}^{+}$cancer stem cell populations has been described to correlate with clinical outcome. Technical points relevant for FACS sorting [7] were also discussed, including the importance of titrating antibody concentration, testing temperature and time of antibody incubations, as well as the Aldefluor assay, which was successfully performed by the participants and quantified by FACS analysis.

After a long and demanding day, dinner at the excellent EMBL canteen was very welcome, and this was followed by our first poster session and refreshments to round things off.

\section{Day Three}

The tissue processing was continued the next day, with organoids being digested to single cells using trypsin/DNase prior to cell staining (anti-Cd45-PECy7, anti-Sca1-PE, antiCd24-FITC and DAPI for Live/Dead segregation) for FACS analysis. Having stained samples, they were analysed with the help of Dr. Paulsen. Although sorting of the different mammary subpopulations was not feasible given the time available, participants were able to identify populations corresponding to basal, luminal estrogen receptor (ER)-negative (ER-) and luminal ER + mammary cells, as well as leukocytes and other non-epithelial populations.

In the afternoon, in between FACS runs, several presentations were given to introduce theoretical background to the following practical work; Dr. Martin Jechlinger gave an overview on 3D culture techniques, discussing historical [8, 9] and technical aspects, such as the use of different matrices and media conditions. The progression of the field towards the cultivation of primary mammary cells $[10,11]$ and the advantages compared to work with cell-lines was discussed. Lastly, Dr. Jechlinger highlighted the importance of analysing tumour progression in the appropriate 3D cellular context and the possibility of doing so with primary mammary cells using approaches that show good correlation with in vivo results from the parental mouse model [12].

Dr. Vivanco discussed mammosphere formation as a tool to enrich for stem cells. In theory, this is a relatively simple assay, however, several aspects should be taken into account to be able to extract the required information: Cells aggregate if cell density is high, affecting clonality and autocrine/ paracrine signals, therefore, keeping low cell density is critical and single cell plating is recommended to assay stem cell properties in vitro. In addition, movement of dishes induces aggregation and it should be kept to the minimum. Once a good single cell suspension has been achieved and cultured, sphere formation should be quantified after a consistent number of days and include sphere size. To demonstrate self-renewal capacity, spheres should be carefully dissociated and replated again at low cell density. The number of cells plated per volume should be reported, as well as the counting method and formation efficiency, represented as number of spheres formed divided by the original number of single cells seeded, including a representative photograph.

Finally, Marta Garcia from EMBL, Heidelberg, introduced basics on histological techniques, ranging from different embedding strategies to standard immune-fluorescence and immune-histochemistry protocols. The talks were followed by a practical session, led by Marta Garcia and Ashna Alladin (EMBL, Heidelberg), on histology techniques introducing paraffin block sectioning, $\mathrm{H} \& \mathrm{E}$ staining and the initial steps of IHC for Ki67 protein on mouse mammary tumour sections.

As a further experimental task on day 3 , the batch of primary tissue samples that had been enzymatically digested overnight was further treated with trypsin to obtain single cell suspensions, which were seeded on collagen coated plates to adhere until further processing for 3D culture on day four.

The beer session following dinner gave us the opportunity to review and discuss another demanding and information rich day.

\section{Day Four}

First thing in the morning of day four, all participants had the opportunity to seed primary mouse mammary cells, retrieved from the collagen coated plates, into 3D matrix. While half of the participants were busy counting, seeding cells and overlaying the solidified BMP-Matrix with media under the guidance of Ashna Alladin and Dr. Jechlinger, the other half proceeded to finish processing the IHC staining. By lunchtime teams had swapped and we were getting ready for an afternoon that focused on imaging technology.

Dr. Stefan Terjung from EMBL, Heidelberg, introduced background and theory on optics and light microscopy, focusing on fluorescent imaging and confocal microscopy. Dr. Terjung also outlined the possibilities to establish joint projects at the Advanced Light Microscopy Facility (ALMF) at EMBL and gave an overview on instruments and the support to the scientific community. His presentation was followed by Dr. Malte Wachsmuth's in-depth introduction to light sheet (SPIM) imaging technology, a technique which is uniquely suited for imaging primary cells in culture, due to its extremely low phototoxicity and speed. 
Following the presentations, participants were split into three groups to rotate between (i) mounting and finishing the IHC process, (ii) joining a tour of the ALMF and (iii) getting a demo on light sheet microscopy. To this end, Ashna Alladin and the team from LUXENDO, led by Dr. Wachsmuth, showed SPIM imaging of 3D culture of mouse mammary gland tumour organoides that express Histone2B-tagged GFP. The image quality and option to follow primary cells over several days without photo-bleaching sparked great interest.

The discussions on yet another very intense and busy day were continued over dinner, which took place downtown in Heidelberg's beautiful old city center. Despite the rather intense program over the last couple of days, a substantial part of the participant crowd used the opportunity to further strengthen newly tied social and scientific bonds over one or two drinks following dinner.

\section{Day Five}

Finally, on Day Five, the wholemounts were ready for visualisation, and time was also available to go back and practice or repeat a particular technique. Participants also used this wrap-up time to take a last look at their 3D cultures. There was a general clamour for more practice at fat pad transplantation - particularly stitching - so a happy couple of hours were spent with scissors, forceps and sutures trying to make the smallest incision possible to identify and clear the fat pad, and then close it up again.

\section{Reaction of the Participants and Forward Look}

As a first time experience, for the course instructors and demonstrators there was a definite learning curve regarding the teaching of the mouse mammary gland techniques to a large group of people within the time limits of the course. Nevertheless, the overall reaction was very positive and we hope that this was due to the thoroughness of preparation given to the participants during the key stages of the processes. In general, the reaction and comments of the participants were excellent with some useful suggestions for the future and they all took new techniques and knowledge back home to teach other members of their own labs. The combination of talks and practicals with plenty of time for open discussions was very much appreciated.

Given the positive and encouraging response we have organised another course for this year (4-9 March 2018, https://www.embl.de/training/events/2018/MAM18-01/) at the same location and EMBO has confirmed their support to the course. We look forward to seeing you next time in Heidelberg!

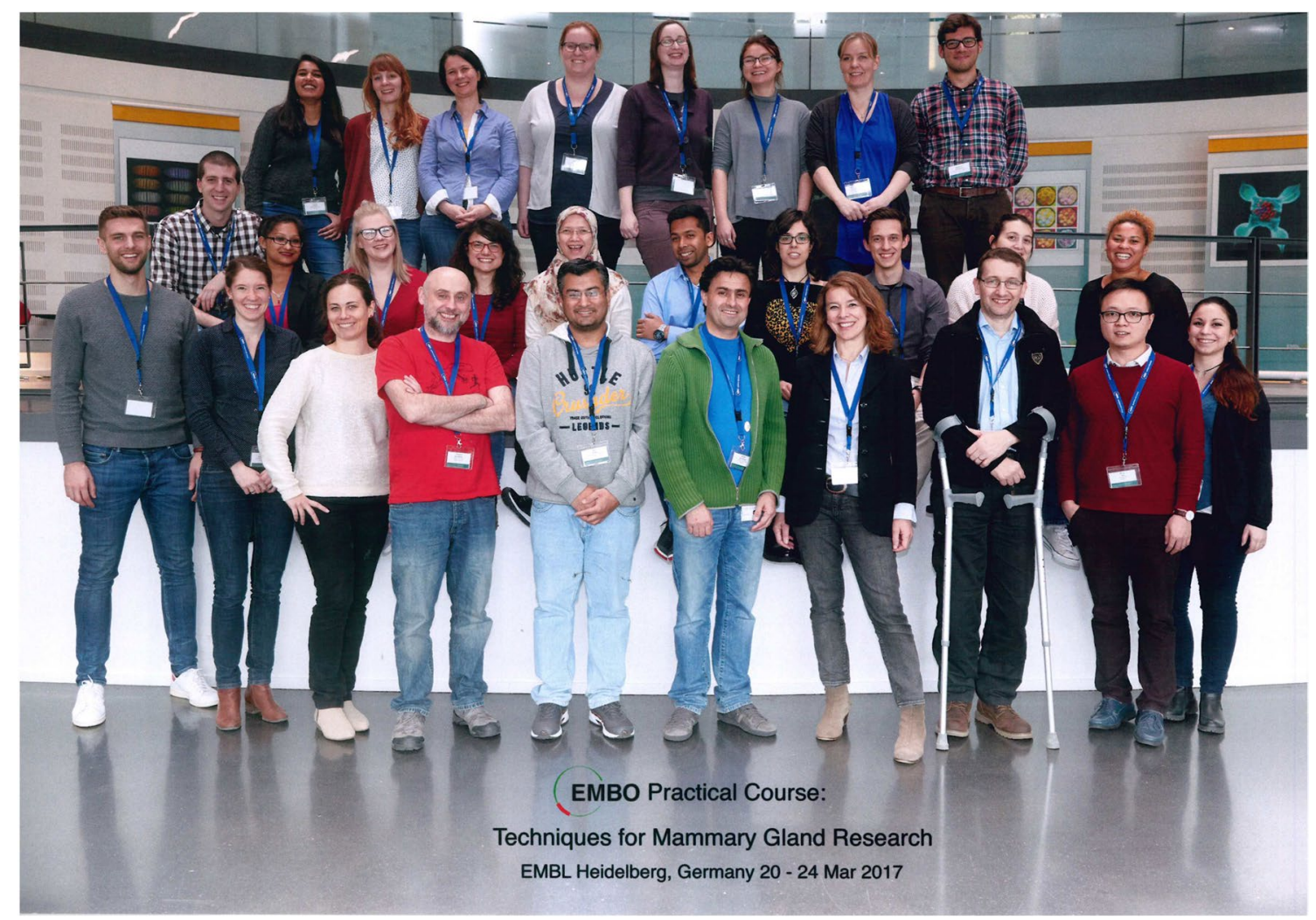


Acknowledgements The course organisers would like to thank Christina Dolt, Yvonne Yeboah and Jaqueline Dreyer for their excellent assistance in organising the course and EMBO for their excellent support; Ashna Alladin and Marta Garcia for help with teaching and demonstrating. Klaus Schmitt for expert animal handling. Dr. Malte Paulsen, Dr. Stefan Terjung and Dr. Malte Wachsmuth for teaching, data analysis and demonstrations.

\section{References}

1. Dontu G, Ince TA. Of mice and women: a comparative tissue biology perspective of breast stem cells and differentiation. J Mammary Gland Biol Neoplasia. 2015;20(1-2):51-62. https:// doi.org/10.1007/s10911-015-9341-4.

2. Smalley MJ. Isolation, culture and analysis of mouse mammary epithelial cells. Methods Mol Biol. 2010;633:139 - 70. https:// doi.org/10.1007/978-1-59745-019-5_11.

3. Smalley MJ, Kendrick H, Sheridan JM, Regan JL, Prater MD, Lindeman GJ, et al. Isolation of mouse mammary epithelial subpopulations: a comparison of leading methods. J Mammary Gland Biol Neoplasia. 2012;17(2):91 - 7. https://doi.org/10.1007/ s10911-012-9257-1.

4. Alexander CM, Puchalski J, Klos KS, Badders N, Ailles L, Kim $\mathrm{CF}$, et al. Separating stem cells by flow cytometry: reducing variability for solid tissues. Cell Stem Cell. 2009;5(6):579 - 83. https ://doi.org/10.1016/j.stem.2009.11.008.

5. Vivanco M. Function follows form: defining mammary stem cells. Sci Transl Med. 2010;2(31):31ps22. https://doi.org/10.1126/scitr anslmed.3001215.
6. Simoes BM, Vivanco MD. Cancer stem cells in the human mammary gland and regulation of their differentiation by estrogen. Future Oncol. 2011;7(8):995-1006. https://doi.org/10.2217/ fon.11.80.

7. Iriondo O, Rabano M, Vivanco MD. FACS Sorting Mammary Stem Cells. Methods Mol Biol. 2015;1293:63-72. https://doi. org/10.1007/978-1-4939-2519-3_3.

8. Barcellos-Hoff MH, Aggeler J, Ram TG, Bissell MJ. Functional differentiation and alveolar morphogenesis of primary mammary cultures on reconstituted basement membrane. Dev. 1989;105(2):223 - 35 .

9. Jechlinger M, Grunert S, Beug H. Mechanisms in epithelial plasticity and metastasis: insights from 3D cultures and expression profiling. J Mammary Gland Biol Neoplasia. 2002;7(4):415 - 32.

10. Jarde T, Lloyd-Lewis B, Thomas M, Kendrick H, Melchor L, Bougaret L, et al. Wnt and Neuregulin1/ErbB signalling extends $3 \mathrm{D}$ culture of hormone responsive mammary organoids. Nat Commun. 2016;7:13207. https://doi.org/10.1038/ncomms13207.

11. Jechlinger M, Podsypanina K, Varmus H. Regulation of transgenes in three-dimensional cultures of primary mouse mammary cells demonstrates oncogene dependence and identifies cells that survive deinduction. Genes Dev. 2009;23(14):1677-88. https://doi. org/10.1101/gad.1801809.

12. Havas KM, Milchevskaya V, Radic K, Alladin A, Kafkia E, Garcia $\mathrm{M}$, et al. Metabolic shifts in residual breast cancer drive tumor recurrence. J Clin Invest. 2017;127(6):2091 - 105. https://doi. org/10.1172/JCI89914. 